\title{
ON CERTAIN VON NEUMANN ALGEBRAS WHICH ARE GENERATED BY PARTIAL ISOMETRIES
}

\author{
CARL PEARCY
}

1. Halmos and McLaughlin recently discovered [2] that a pair of arbitrary (bounded) operators on Hilbert space are unitarily equivalent if and only if a certain pair of associated partial isometries are also unitarily equivalent; thus they concluded that the unitary equivalence problem for the class of partial isometries is extremely difficult. The purpose of this present note is to show that partial isometries can be unpleasant in other ways. More specifically:

TheOREM. There exists a partial isometry $P$ (resp. $Q)$ which generates a von Neumann algebra of type $\mathrm{II}_{1}\left(\right.$ resp. $\left.\mathrm{II}_{\infty}\right){ }^{1}$

(This result is evidence that partial isometries are intrinsically more complicated than isometries, because it follows easily from the known structure theory of isometries that an isometry must generate a von Neumann algebra of type I.)

The proof of the theorem consists of two observations which are interesting in themselves, and we record them as lemmas.

Lemma 1. If $A$ is an invertible operator on a Hilbert space $\mathfrak{H}$, and $A$ generates the v.N. algebra $\mathrm{R}$, then there is a partial isometry $P$ on the Hilbert space $\mathcal{H} \oplus \mathcal{H C}$ which generates the v.N. algebra $\mathrm{T}$ consisting of all $2 \times 2$ matrices over R. Furthermore, the type of the v.N. algebra $\mathrm{T}$ is the same as that of $\mathrm{R}$.

Proof. We can assume that $A$ satisfies $\|A\|<1$, and we introduce the notation $M\left(B_{11}, B_{12}, B_{21}, B_{22}\right)$ for the $2 \times 2$ operator matrix

$$
\left(\begin{array}{ll}
B_{11} & B_{12} \\
B_{21} & B_{22}
\end{array}\right)
$$

acting on the Hilbert space $\mathcal{H} \oplus \mathcal{H}$ in the usual fashion. Let $P=M(A, 0, T, 0)$, where $T \in \mathrm{R}$ is the positive square root of $1-A^{*} A$. ( 1 is the identity operator on $\mathcal{F}$.) Computation shows that $P^{*} P$ is the matrix $E=M(1,0,0,0)$, i.e., that $P$ is a partial isometry. ( $P$ is, in fact, the partial isometry set forth by Halmos which played a role

Received by the editors February 13, 1963.

1 The von Neumann (v.N.) algebra generated by an operator is the smallest v.N. algebra which contains the operator. The results of this note remain true whether or not one assumes that a v.N. algebra always contains the scalar operators. 
in the above mentioned discovery of Halmos and McLaughlin.) Since $E P$ is the matrix $M(A, 0,0,0)$, it is obvious that the v.N. algebra T contains all matrices of the form $M(S, 0,0,0)$, where $S \in \mathbf{R}$. Calculation shows that $P P^{*}=\left(A A^{*}, A T, T A^{*}, 1-A^{*} A\right)$ and also that $P P^{*}+M\left(A A^{*}, 0,0,0\right)-\left(P P^{*} E+E P P^{*}\right)=M\left(0,0,0,1-A^{*} A\right)$. Hence T contains all matrices of the form $M(0,0,0, S)$, where $S$ is in the v.N. algebra generated by the invertible, positive definite operator $1-A^{*} A$. Thus in particular $F=M(0,0,0,1)$ and $M\left(0,0,0, T^{-1}\right)$ are in $T$. Now

$$
M\left(0,0,0, T^{-1}\right)\left(P P^{*} E\right) M\left(A^{*-1}, 0,0,0\right)=M(0,0,1,0)=U
$$

and thus $\mathrm{T}$ contains the set of matrix units $E, F, U, U^{*}$. It follows easily that $\mathrm{T}$ contains every matrix $M\left(B_{11}, B_{12}, B_{21}, B_{22}\right)$, where the $B_{i j} \in \mathbf{R}$. It is also easy to verify that the collection of all such matrices is a v.N. algebra (it is weakly closed), so that $T$ consists exactly of all $2 \times 2$ matrices with entries from $R$. The fact that type is preserved in the passage from $R$ to $T$ was proved by Berberian in [1]. (This means, of course, that if $R$ is of type I finite, $\mathrm{I}_{\infty}, \mathrm{II}_{1}, \mathrm{II}_{\infty}$, or III, then $T$ is of the same type.)

Lemma 2. There exists an invertible operator $A$ (resp. B) on a Hilbert space $\mathfrak{H C}$ such that the v.N. algebra generated by $A$ is a factor of type $\mathrm{II}_{1}$ (resp. $\mathrm{II}_{\infty}$ ).

Proof. We show that some factors of types $\mathrm{II}_{1}$ and $\mathrm{II}_{\infty}$ set forth by Murray and von Neumann in [4] have single invertible generators. In particular, we follow the presentation of Kadison [3]. The constructions involve certain measure spaces $(S, \delta, \mu)$. To construct a factor of type $\mathrm{II}_{1}$, we take $(S, \delta, \mu)$ to be the unit circle under Lebesgue measure, and we consider the abelian group $G$ of measure preserving transformations of $S$ which is generated by a rotation of the circle through an irrational angle. Let $\Re=L_{2}(S, \mu)$, and for each element $g \in G$, consider the operators $U_{g}$ and $L_{g}$ on $\mathcal{K}$ defined by $\left(U_{o}(f)\right)(x)$ $=f(g(x))$ and $L_{g}(f)=g f$, respectively. Then each $U_{\theta}$ is unitary, each $L_{o}$ is normal, the $U_{o}$ are all mutually commuting, and the $L_{g}$ are all mutually commuting. Let $\mathfrak{H C}$ be the direct sum of countably many copies of $\mathcal{K}$. We define some operators on $\mathcal{H}$ which consist of infinite matrices with rows and columns indexed by $G$. For each $g \in G$, let $V_{0}$ be the matrix whose only entry in the $h$ column is $U_{0}$ in the $g h$ row, and let $N_{g}$ be the diagonal matrix with each diagonal entry equal to $L_{g}$. A calculation shows that each $V_{g}$ is a unitary operator on $\mathcal{H C}$ and that the v.N. algebra $V$ which the collection $\left\{V_{o} \mid g \in G\right\}$ generates is abelian. It is obvious that the v.N. algebra $Y$ which the col- 
lection $\left\{N_{o} \mid g \in G\right\}$ generates is also abelian. The v.N. algebra $\mathbf{R}$ which the pair $(V, Y)$ generates is known to be a factor of type $\mathrm{II}_{1}$ [4]. A single invertible generator $A$ for $\mathbf{R}$ is obtained as follows. One knows from [5] that there exists a single Hermitian generator $D$ for the abelian algebra $\mathbf{Y}$, and there exists a single Hermitian generator $C$ for the abelian algebra V. Furthermore, $C$ can be taken to be invertible. For a sufficiently small positive scalar $\alpha, C+i \alpha D=A$ will be invertible, and clearly $A$ will generate the type $\mathrm{II}_{1}$ factor $\mathrm{R}$. To obtain an invertible operator which generates a factor of type $\mathrm{II}_{\infty}$, one proceeds exactly as above, except that the measure space $(S, \delta, \mu)$ is taken to be the real line under Lebesgue measure, and the abelian group $G$ is taken to be the group of rational translations of the line. (Verification of the fact that the corresponding algebra $V$ remains abelian requires another calculation.)

Remark. (Added in proof). Recently, N. Suzuki and T. Saitô obtained in the note [On the operators which generate continuous von Neumann algebras, Tôhoku Math. J. 15 (1963), 277-280] results similar to Lemma 2 above.

\section{BIBLIOGRAPHY}

1. S. K. Berberian, $N \times N$ matrices over an $\mathrm{AW}^{*}$-algebra, Amer. J. Math. 80 (1958), $37-44$.

2. P. R. Halmos and J. E. McLaughlin, Partial isometries, Pacific J. Math. 13 (1963), 585-596.

3. R. V. Kadison, Theory of operators. II. Operator algebras, Bull. Amer. Math. Soc. 64 (1958), 61-85.

4. F. J. Murray and J. von Neumann, On rings of operators, Ann. of Math. (2) 37 (1936), 116-229.

5. J. von Neumann, Über Funktionen von funktional Operatoren, Ann. of Math. 32 (1931), 191-226.

Humble Oil and Refining Company 